\title{
DIFERENCIAÇÃo FLORAL, ALTERNÂNCIA DE PRODUÇÃO E USO DE ÁCIDO GIBERÉLICO EM TANGERINEIRA 'MONTENEGRINA' (Citrus deliciosa Tenore) ${ }^{1}$
}

\author{
ANA MARÍA RAMOS-HURTADO²; OTTO CARLOS KOLLER ${ }^{3}$; JORGE DE ARAÚJO MARIATH4 ; IVAR ANTONIO \\ SARTORI $^{5}$; SERGIOMAR THEISEN ${ }^{5}$; BERNADETE REIS ${ }^{2}$
}

\begin{abstract}
RESUMO - A alternância de produção é um problema na cultura da tangerina 'Montenegrina' (Citrus deliciosa Ten.). Objetivou-se testar o uso do ácido giberélico e épocas de aplicação na inibição floral e conseqüente diminuição da alternância. O experimento foi executado no período de abril de 2004 a agosto de 2005, no município de Eldorado do Sul, Estado do Rio Grande do Sul, em pomar de 20 anos com plantas enxertadas sobre Poncirus trifoliata Raf. Noventa e seis plantas uniformes, em alternância de produção e com poucos frutos foram selecionadas e arranjadas em delineamento de blocos ao acaso, com quatro repetições e duas plantas úteis por parcela. Os tratamentos foram arranjados em esquema fatorial com quatro concentrações de $\mathrm{AG}_{3}(0 ; 20 ; 40$ e $60 \mathrm{mg} / \mathrm{L})$ e três épocas de aplicação (30 de abril, 20 de maio e 10 de junho), nos anos de 2004 e 2005. Nenhum tratamento inibiu a floração, nem afetou o número e a massa dos frutos colhidos. Observações histológicas de cortes longitudinais de gemas, feitas quinzenalmente, evidenciaram que a diferenciação de gemas de flor, perceptível pela emissão das primeiras sépalas, só ocorre na primeira quinzena do mês de agosto, indicando que a indução da diferenciação floral deve verificar-se em julho, bem depois das aplicações de $\mathrm{AG}_{3}$ feitas nesta pesquisa.
\end{abstract}

Termos para indexação: Citrus deliciosa, alternância de produção, diferenciação floral, ácido giberélico

\section{FLOWER BUD DIFFERENTIATION, ALTERNATE BEARING AND APPLICATION OF GIBBERELLIC ACID ON 'MONTENEGRINA' TANGERINE TREES (Citrus deliciosa Tenore).}

\begin{abstract}
Alternate bearing is an important problem of 'Montenegrina' mandarins (Citrus deliciosa Ten.). The aim of this work was to supress flower bud formation in 'off' or light crop years and thereby reduce biennal bearing. The experiment was carried out from april 2004 to august 2005, in Eldorado do Sul - RS, on a twenty-year-old orchard with trees budded onto Poncirus trifoliata Raf. Ninety six trees were rated as having very light fruit load. A randomized blocks experimental design with $3 \times 4$ factorial scheme with two plants per plot and four replications was used. The trees were sprayed with $0,20,40$ e $60 \mathrm{mg} \cdot \mathrm{L}^{-1} \mathrm{GA}_{3}$. The foliar applications were made on April 30 , May 20 and June 10 , at the two years. $\mathrm{GA}_{3}$ did not suppress flowering and the number of fruits per tree and yield were not affected by the treatments. Histological studies on bud longitudinal sections, realized every fifteen days in 2005, between April 30 and August 30, showed that the period of flower bud differentiation, with emission of the first sepals, only occurs on the first half of August. This indicates that the induction of floral diferenciation may occur in july, after the timings of $\mathrm{GA}_{3}$ spraying in this research.
\end{abstract}

Index terms: Citrus deliciosa, biennal bearing, flower bud differentiation, gibberellic acid.

\section{INTRODUÇÃO}

As tangerinas são o segundo grupo de frutos cítricos em importância na citricultura mundial. Os principais países produtores são: China, Espanha e Japão, sendo que o Brasil está situado como o quarto produtor, apresentando uma área cultivada superior a $65 \mathrm{mil}$ ha e uma produção de 1,3 milhão de toneladas por ano (Anuário Brasileiro da Fruticultura, 2005). Os principais Estados produtores de tangerinas são, pela ordem: São Paulo, Rio Grande do Sul, Paraná, Bahia e Minas Gerais, que perfazem 90\% da área total plantada (Amaro $\&$ Caser, 2003). Entre as cultivares plantadas no Estado do Rio Grande do Sul, salienta-se a tangerineira 'Montenegrina' (Citrus deliciosa Tenore), que ocupa a terceira parte da superfície plantada (Rodrigues et al., 1998).

Característica comum nos citros, especialmente nas tangerineiras, a 'Montenegrina' apresenta uma produção irregular ao longo dos anos, devido ao alto grau de alternância. Esse comportamento caracteriza-se por um ano de excessiva carga de frutos, seguido de outro ano com produção muito baixa ou nula. Nos anos de florescimento excessivo, os frutos são de baixa qualidade, pequenos, de coloração deficiente, aguados e ácidos (Koller, 1994).

Entre as causas da alternância de produção, citam-se fatores exógenos: condições climáticas, tais como temperaturas favoráveis ou desfavoráveis à frutificação e geadas ou secas (Hield \& Hilgerman, 1969), ataque de pragas e doenças (Wheaton, 1986) e fatores endógenos: carência de carboidratos (Goldschmidt \& Golomb, 1982; Souza, 1990) e desbalanços hormonais (Moss, 1971). Tem sido observado que quando ocorre a alternância de produção, provocada por um ou mais desses fatores, as plantas tendem a permanecer em alternância por tempo indefinido, sendo necessária a utilização de práticas culturais adequadas para que as árvores voltem a florescer e frutificar com regularidade.

Diversos métodos para resolver esse problema, como: poda (Koller, 1994; Sartori, 2005); raleio químico e manual (Marodin, 1986; Souza, 1990); controle da floração com fitorreguladores (Monselise \& Halevy, 1964; Moss, 1971; Agustí, 2000), têm sido estudados e usados de forma crescente nas principais regiões citrícolas do mundo. No Brasil, eles ainda são pouco utilizados e no Estado do Rio Grande do Sul, onde a maioria das propriedades é de exploração familiar, o raleio de frutos é realizado manualmente. Se o preço for compensador, os frutos raleados podem ser vendidos a indústrias que extraem óleos essenciais da casca verde (Schwarz, 1989; Schwarz et al., 1992).

O raleio manual, entretanto, é muito dispendioso em mão-deobra (Schwarz, 1989) e, sendo realizado quando os frutos já estão com certo desenvolvimento ( 2 a $3 \mathrm{~cm}$ de diâmetro), estes já consumiram boa parte das reservas da planta. Assim sendo, para

\footnotetext{
1 (Trabalho 50-2006). Recebio: 26-04-2006. Aceito para publicação: 06-10-2006.

${ }^{2}$ Eng $^{\mathrm{a}}$ Agra , aluna de mestrado do Programa de Pós-Graduação em Fitotecnia da UFRGS. Av. Bento Gonçalves 7712,

Bairro Agronomia, Cx. P 776, CEP 91591970 Porto Alegre - RS. Email: anarequipa@yahoo.com.br

Dr. Prof. Convidado da Faculdade de Agronomia da UFRGS, Bolsista 1A do CNPq. Endereço Rua Largo Setembrina, 126

Viamão - RS.CEP 94415-400

${ }^{4}$ Dr. Prof. Titular do Depto. de Botânica da UFRGS, Av. Bento Gonçalves, 9500, Bairro Agronomia, CEP 9.500 91501-970, Porto Alegre - RS. E-mail: jorge.mariath@ufrgs.br

${ }^{5}$ Eng $^{\circ}$ Agr $^{\circ}$ Dr. em Fitotecnia, Professor Substituto de Fruticultura - UFRGS. Departamento de Fruticultura e Silvicultura. Av Bento Gonçalves, 7712. Bairro Agronomia. E mail: ivar@ufrgs.br Fone: (51) 3316-6020.
} 
que se obtenham bons resultados em melhoria da qualidade dos frutos e quebra da alternância de produção, pode ser necessário ralear 60 a $80 \%$ dos frutos, diminuindo a massa da produção total de frutos por planta (Schwarz, 1989).

Aplicações de reguladores de crescimento, especialmente, mas não exclusivamente de $\mathrm{AG}_{3}$ em citros, têm melhorado a produtividade e a qualidade dos frutos, sendo já utilizadas em pomares comerciais há alguns anos. Os reguladores de crescimento têm sido usados para alterar a floração, melhorar a fixação, raleio e abscisão de frutos (Berhow, 2000).

Assim sendo, o raleio químico de flores, através de pulverizações com fitorreguladores, poderia ser uma alternativa de execução rápida para quebrar a alternância de produção, possivelmente sem prejudicar a produção comercial de frutos, principalmente em épocas e regiões em que não for vantajoso o raleio manual, devido ao baixo valor dos frutos raleados, destinados às indústrias de óleos essenciais.

É conhecido que o florescimento em plantas cítricas é precedido por um período de transformação das gemas vegetativas em reprodutivas através de estímulos e da paralisação do crescimento vegetativo, seja por seca, seja por frio. A primeira mudança que ocorre é o aumento da atividade mitótica entre a zona central e a periférica do meristema; a segunda mudança caracteriza-se pelo achatamento do meristema, após a iniciação de cada primórdio de sépala. Gemas achatadas com iniciação das sépalas representam a padronização de gemas florais (Randhawa \& Dinsa, 1947).

Estudos feitos na Flórida, EUA, indicam que o início da diferenciação das gemas florais em laranjeiras e pomeleiros acontece em janeiro. As tangerineiras iniciam a diferenciação uma ou duas semanas mais tarde, provavelmente por ser sua floração mais tardia (Albrigo, 1997).

Sabe-se que as giberelinas $\left(\mathrm{AG}_{3}\right)$, além de promoverem o crescimento vegetativo, têm efeito inibitório sobre a diferenciação floral, evitando ou diminuindo a produção de flores. Isso fol evidenciado por diversas pesquisas com aplicação exógena de GA em citros (Monselise \& Halevy, 1964; Moss \& Bevington, 1977; Guardiola et al., 1982), mas as aplicações devem ser feitas na época e na concentração adequadas, sendo crítico o momento da aplicação (Guardiola, 1994). Moss \& Bevington (1977), na Austrália, conseguiram reduzir a floração da laranjeira 'Valência' até em $25 \%$, aplicando $25 \mathrm{mg} . \mathrm{L}^{-1}$ de $\mathrm{AG}_{3}$ durante os meses de abril e maio.

Entretanto, existem controvérsias quanto ao momento mais apropriado para aplicar o ácido giberélico, com o objetivo de inibir a diferenciação floral. Para Monselise e Halevy (1964), pulverizações com $\mathrm{AG}_{3}$ devem ser feitas durante a indução floral, bem antes que as gemas iniciem a brotação, pois se estas estiverem diferenciadas, não sofrerão mais a ação do $\mathrm{AG}_{3}$.

Segundo Agustí \& Almela (1991), são sugeridos dois momentos para a aplicação do $\mathrm{AG}_{3}$ : o primeiro no estádio de indução floral, outono e início de inverno, não existindo nenhum indicador fisiológico que possa identificar esse estádio; e o segundo na ocasião da iniciação floral, que daria melhor resultado do que o primeiro (Agustí, 2000). Porém este último momento requer identificação do adequado desenvolvimento das brotações, porque depois que elas ultrapassam os três milímetros as gemas de flor já estão prontas, as pétalas e outros órgãos estão se desenvolvendo, e aplicações de $\mathrm{AG}_{3}$ já não são mais eficazes (Lord \& Eckard, 1987).

Para controlar o excesso de flores em laranjeiras-de-umbigo, na Espanha, Agustí e Almela (1991) recomendam a pulverização foliar com $10 \mathrm{mg} . \mathrm{L}^{-1}$ de $\mathrm{AG}_{3}+15 \mathrm{ppm}$ de 2,4-D, em fins de novembro ou início de dezembro (Hemisfério Norte). No Brasil,Castro (2001) recomenda aplicações de $15 \mathrm{mg} \cdot \mathrm{L}^{-1}$ de $\mathrm{AG}_{3}$ só ou em combinação com 2,4-D, nos meses de julho e agosto, quando as brotações ainda não atingiram três milímetros de comprimento. Nessa época, elas redistribuem a brotação e reduzem a floração com a mesma intensidade que as aplicações efetuadas durante o repouso invernal.
Os objetivos deste trabalho foram de identificar a época da diferenciação floral e testar concentrações e épocas de aplicação de $\mathrm{AG}_{3}$, para tentar neutralizar ou diminuir a alternância de produção da tangerineira 'Montenegrina', mediante a inibição da diferenciação floral, nas condições edafoclimáticas do Estado do Rio Grande do Sul.

\section{MATERIAL E MÉTODOS}

O trabalho foi conduzido durante o período de abril de 2004 a agosto de 2005, no município de Eldorado do Sul- RS, km. 146 da BR290, na latitude $30^{\circ} 05^{\prime} 27^{\prime \prime} \mathrm{S}$ e longitude 51\%40'18' W e uma altitude aproximada de $50 \mathrm{~m}$

Foi utilizado um pomar de tangerineiras 'Montenegrina' (Citrus deliciosa Tenore), de aproximadamente 20 anos de idade, enxertadas sobre o Poncirus trifoliata Raf. e plantadas em espaçamento de $5 \times 2,5 \mathrm{~m}$. O solo da área experimental é classificado como Podzólico Vermelho-Escuro, de textura franco-argilosa. O clima é do tipo Cfa, segundo a classificação de Köeppen, subtropical sem estação seca definida. Foram selecionadas 96 plantas uniformes, que se encontravam em alternância, com poucos frutos. O delineamento experimental adotado foi o de blocos casualizados, em esquema fatorial 3 x 4, com quatro repetições, testando-se 3 épocas de aplicação de ácido giberélico $\left(\mathrm{AG}_{3}\right)$ : 30 de abril, 20 de maio e 10 de junho, e 4 concentrações de $\mathrm{AG}_{3}: 0 ; 20 ; 40$ e $60 \mathrm{mg} . \mathrm{L}^{-1}$. Cada bloco foi composto de 12 tratamentos, e cada parcela foi constituída de duas plantas, utilizando-se de duas árvores como bordaduras entre cada tratamento na linha de plantas.

O ácido giberélico foi aplicado no outono de 2004 e novamente no outono de 2005, sob a forma de produto comercial ProGibb (100 g/ $\mathrm{kg} \mathrm{de} \mathrm{AG}_{3}$ ). Utilizou-se um pulverizador motorizado marca Hatsuta, com capacidade para 400 litros, acionado por um trator Massey Ferguson 275, com duas pistolas de pulverização com jato cônico. Foram utilizados ao redor de 8 litros de solução por planta, chegando a ponto de início de escorrimento.

Para realizar análises histológicas e verificar a época de diferenciação floral, no ano de 2004, a cada 15 dias, desde 30 de abril até 15 de junho, das plantas de cada parcela, foram coletados ramos frutíferos, que haviam brotado no verão. Em 2005, prevendo que as plantas do experimento entrariam em alternância de produção (não floresceriam), os ramos frutíferos foram coletados desde 15 de junho até 30 de agosto, de 5 árvores do mesmo pomar, mas que não faziam parte do experimento, e que estavam entrando em ano em que deveriam florescer intensamente. Após a coleta e identificação dos ramos, suas folhas foram cortadas com uma tesoura de poda, e cada ramo foi seccionado em segmentos de 7 a $10 \mathrm{~mm}$ de comprimento, cada qual com uma gema. Imediatamente, esses segmentos foram colocados em frascos de $50 \mathrm{ml}$ que continham um meio fixador de 'Karnowsky' (formulação em volume: $100 \mathrm{ml} . \mathrm{L}^{-1}$ de glutaraldeído a 250mg. $\mathrm{L}^{-1}+250 \mathrm{ml} \cdot \mathrm{L}^{-1}$ de paraformaldeído a $80 \mathrm{mg} . \mathrm{L}^{-1}+500 \mathrm{ml} \cdot \mathrm{L}^{-1} \mathrm{de}$ tampão fosfato $0,2 \mathrm{M}+150 \mathrm{ml} \cdot \mathrm{L}^{-1}$ de água destilada). As gemas permaneceram, no mínimo, uma semana na solução fixadora até sua posterior avaliação microscópica.

Para a avaliação microscópica, foram selecionadas ao acaso 10 gemas de cada tratamento. Com o auxílio de uma pinça, os segmentos de ramo foram colocados em placas de Petri, levados para um microscópio estereoscópico de 10 aumentos. Com ajuda de uma pinça fina para segurar os segmentos de ramos, procedeu-se a dissecação das gemas realizando cortes longitudinais com um bisturi. Os cortes longitudinais eram colocados entre lâmina e lamínula e imediatamente levadas para observação em microscópio de campo claro, observados em 10 e 20 aumentos e, assim, verificado se apresentavam gemas vegetativas ou se o processo de diferenciação floral já havia iniciado. Os registros fotomicrográficos foram realizados no microscópico Leica DMR, com auxílio de câmara digital Nikon Coolpix 950, e as imagens, processadas no programa Adobe Photoshop. 
TABELA 1 - Número de flores emitidas por 100 gramas de massa verde de ramos frutíferos de tangerineira 'Montenegrina' (Citrus deliciosa Tenore), em função de quatro concentrações e três épocas de aplicação de $\mathrm{AG}_{3}$. Eldorado do Sul - RS, 2005.

\section{Número de flores $/ 100 \mathrm{~g}$ de ramos frutíferos}

Tratamentos

Época de aplicação de $\mathbf{A G}_{3}$

\begin{tabular}{lllll} 
& Abril & Maio & Junho & Média \\
\hline Testemunha & $188,08 \mathrm{~ns}$ & $203,55 \mathrm{~ns}$ & $192,51 \mathrm{~ns}$ & $194,72 \mathrm{~ns}$ \\
$20 \mathrm{mg} / \mathrm{LAG}_{3}$ & 137,59 & 167,22 & 242,37 & 182,56 \\
$40 \mathrm{mg} / \mathrm{LAG}_{3}$ & 219,38 & 197,83 & 148,47 & 188,56 \\
$60 \mathrm{mg} / \mathrm{LAG}_{3}$ & 257,37 & 253,81 & 211,27 & 240,82 \\
\hline Média & $200,61 \mathrm{~ns}$ & 205,61 & 198,66 & \\
\hline
\end{tabular}

CV

$20,20 \%$

ns: não houve diferença estatística entre os tratamentos, ao nível de $5 \%$ de probabilidade.

TABELA 2.- Número de frutos produzidos por tangerineira 'Montenegrina' (Citrus deliciosa Tenore), em decorrência de quatro concentrações e três épocas de aplicação de $\mathrm{AG}_{3}$. Eldorado do Sul - RS. 2005.

\begin{tabular}{lllll}
\hline & \multicolumn{4}{c}{ Número total de frutos/planta } \\
\cline { 2 - 5 } Tratamentos & \multicolumn{4}{c}{ Época de aplicação de $\mathbf{A G}_{\mathbf{3}}$} \\
\cline { 2 - 5 } & Abril & Maio & Junho & Média \\
\hline Testemunha & $726,37 \mathrm{~ns}$ & $672,50 \mathrm{~ns}$ & $696,62 \mathrm{~ns}$ & $698,50 \mathrm{~ns}$ \\
$20 \mathrm{mg} / \mathrm{L} \mathrm{AG}_{3}$ & 704,00 & 853,12 & 596,87 & 718,00 \\
$40 \mathrm{mg} / \mathrm{L} \mathrm{AG}_{3}$ & 676,12 & 631,00 & 687,75 & 664,96 \\
$60 \mathrm{mg} / \mathrm{L} \mathrm{AG}_{3}$ & 747,00 & 778,12 & 690,75 & 738,63 \\
\hline Média & $713,38 \mathrm{~ns}$ & 733,69 & 668,00 & \\
\hline CV & & \multicolumn{3}{c}{$\mathbf{1 4 , 5 0 \%}$} \\
\hline
\end{tabular}

ns: não houve diferença estatística entre os tratamentos, ao nível de $5 \%$ de probabilidade.

TABELA 3. Porcentagem de gemas vegetativas e gemas diferenciadas de árvores alternantes de tangerineira 'Montenegrina' Eldorado do Sul - RS. 2005.

\begin{tabular}{lcccccc}
\hline \multirow{2}{*}{ Gemas } & \multicolumn{6}{c}{ \% Gemas vegetativas e diferenciadas } \\
\cline { 2 - 7 } & \multicolumn{6}{c}{ Datas de coleta } \\
\cline { 2 - 7 } & $15-06$ & $30-06$ & $15-07$ & $30-07$ & $15-08$ & $30-08$ \\
\hline Gemas diferenciadas & 0 & 0 & 2 & 14 & 36 & 30 \\
Gemas vegetativas & 100 & 100 & 98 & 86 & 64 & 70 \\
\hline
\end{tabular}

Para a avaliação do índice de florescimento, antes das pulverizações com $\mathrm{AG}_{3}$ em abril de 2004, em cada planta útil, foram marcados dois galhos portadores de ramos frutíferos, situados a aproximadamente $1,5 \mathrm{~m}$ de altura, nos quadrantes leste e oeste das linhas de plantas. Em 24 de setembro de 2004, foram podados e coletados todos os ramos frutíferos que haviam sido formados no verão/outono. Esses ramos, com e/ou sem brotações florais, foram identificados, acondicionados em sacos plásticos e no mesmo dia eles foram pesados, contadas as flores e os botões florais presentes, e depois foi determinada a relação do número de flores/100 $\mathrm{g}$ de massa vegetal verde. Dos dias 15 a 17 de agosto de 2005, todos os frutos produzidos por planta foram colhidos, classificados em três calibres comerciais, contados e pesados.

Todos os dados obtidos foram submetidos à análise de variância, segundo modelo de delineamento de blocos ao acaso, com esquema fatorial, e utilizando o F-teste, ao nível de 5\% de probabilidade, para testar a significância das diferenças. Para avaliar efeitos de doses de $\mathrm{AG}_{3}$ foram realizadas análises de regressão.

\section{RESULTADOS E DISCUSSÃO}

Os resultados da contagem de flores emitidas por $100 \mathrm{~g}$ de massa de ramos, 3 a 5 meses após a aplicação dos tratamentos, são apresentados na Tabela 1. Não houve diferenças significativas entre épocas de aplicação, nem entre concentrações de $\mathrm{AG}_{3}$ sobre o número de flores. Além disso, através de análise de regressão, verificou-se que não existiu qualquer tendência de efeito de raleio de flores.

Estes dados estão em concordância com os resultados obtidos por Sartori (2005), em tangerineira 'Montenegrina' e por Gallash (1978), na Austrália, em laranjeira 'Valência', porém diferem de Moss \& Bevington (1977), também na Austrália, que conseguiram inibir a floração da laranjeira 'Valência' com aplicações de $\mathrm{AG}_{3}$, realizadas 

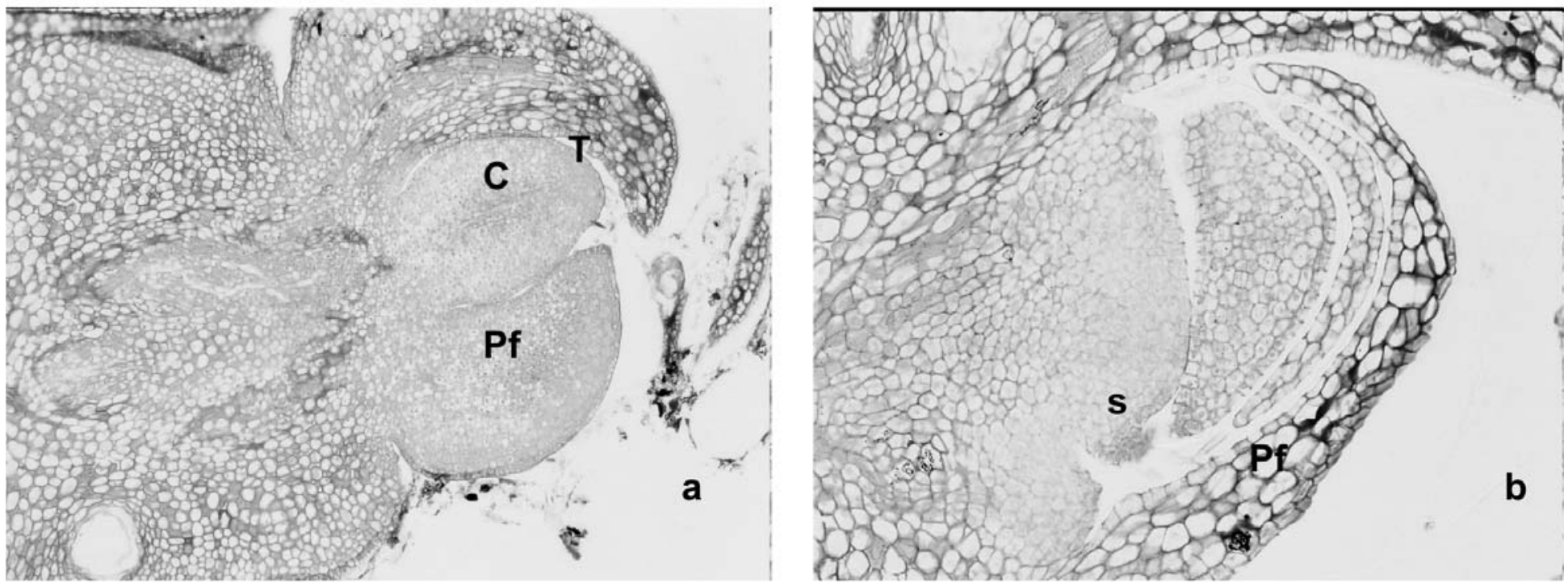

FIGURA 1 - Fotomicrografias de seção longitudinal de gemas de tangerineira 'Montenegrina': a) gema vegetativa cônica, onde C = corpo; T = túnica $; \mathrm{Pf}=$ Primórdios foliares (terceira semana de julho de 2005). 10X. b) gema diferenciada com início de emissão de sépalas, onde $\mathrm{s}=$ sépalas; $\mathrm{Pf}=$ primórdio foliar (15-08-05). 20X

nos meses de abril e maio, prévias a uma floração excessiva.

Com relação ao número total de frutos, produzidos pelas plantas submetidas aos tratamentos com aplicação de $\mathrm{AG}_{3}$ e sua interação com as épocas de aplicação, não houve diferenças significativas entre os tratamentos (Tabela 2). Os resultados obtidos corroboram, pois, que a aplicação de $\mathrm{AG}_{3}$, não inibiu a floração nem a frutificação da tangerineira 'Montenegrina', e por conseguinte não foi útil para quebrar a alternância de produção.

Em relação à evolução anatômica, nas observações microscópicas realizadas em gemas coletadas quinzenalmente, desde 15 de abril a 15 de junho de 2004, não foi constatado nenhum indício de diferenciação floral, pois todas conservaram o formato cônico de gemas vegetativas (Schneider, 1968), como pode ser observado na Figura 1.

Em 2005, quando as gemas foram coletadas desde 15 de junho até 30 de agosto, só foram encontradas as primeiras gemas diferenciadas, com emissão de sépalas, a partir da terceira semana do mês de julho (Tabela 3). O número de gemas florais diferenciadas aumentou nas coletas seguintes, atingindo sua maior porcentagem na terceira semana do mês de agosto.

Com base nesses dados e no tempo de duas a três semanas, que, segundo Erickson (1968), decorre entre a indução e a diferenciação floral, pode-se estabelecer que, em 2005, a época da diferenciação floral ocorreu entre a segunda e a terceira semana do mês de agosto, e a indução floral, duas a três semanas antes, aproximadamente na terceira e quarta semanas de julho.

Por sua vez, verificou-se que, em 2004, as árvores encontravam-se em plena floração ao final de setembro e início de outubro. Considerando que o intervalo de tempo entre o momento da diferenciação floral (emissão de sépalas) e a plena floração é de aproximadamente dois meses (Tadeo et al., 2003), e entre a indução floral e a diferenciação, de duas a três semanas, Ayalon \& Monselise, 1959, citados por Pereira et al. 2003, afirmam então, que a indução floral, em 2004, deve ter acontecido no início ou na metade do mês de julho. Variações na época da diferenciação floral, de um ano para outro, também foram observadas por Iqbal \& Karacali (2004), em tangerineira 'Satsuma', e por Pereira et al. (2003), em laranjeira 'Pêra'.

Isso indica que, nesta pesquisa, $\mathrm{o}_{\mathrm{AG}_{3}}$ pode não ter exercido efeito de inibição da floração, nas aplicações feitas em 2004, porque elas foram realizadas com muita antecedência ao momento da indução floral (Monselise e Halevy, 1964 e Agustí \& Almela, 1991). Assim, seria conveniente fazer testes de aplicação entre a primeira e quarta semana do mês de julho, ocasião em que é maior a probabilidade de atingir o momento crítico de máxima resposta.

\section{CONCLUSÕES}

1) No local, épocas e concentrações de ácido giberélico aplicadas, não se consegue inibir a diferenciação floral nem controlar a alternância de produção da tangerineira 'Montenegrina'.

2) A diferenciação de gemas de flor verifica-se na primeira quinzena do mês de agosto, e a indução da diferenciação floral deve acontecer no mês de julho.

\section{REFERÊNCIAS}

AGUSTÍ, M. Citricultura. Madrid: Mundi-Prensa, 2000. 416p.

AGUSTÍ, M.; ALMELA, V. Aplicación de fitorreguladores em citricultura. Barcelona: Editorial Aedos, 1991.261p.

ALBRIGO, L..G. Induction and flowering processes: Florida perspective. Lake Alfred, FL: Citrus Research and Education Center, 1997. Disponível em <www.fcprac.ifas.ufl.edu/>. Acesso em: 15 jun. 2005.

AMARO, A.A.; CASER, D.V. Diversidade do mercado de tangerinas. Informações Econômicas, São Paulo, v. 33, n. 12 p. 51-67, 2003.

ANUÁRIO BRASILEIRO DA FRUTICULTURA. Santa Cruz do Sul: Gazeta, 2005. 136 p.

BERHOW, M.A. Effect of early plant growth regulator treatments on flavonoid levels in grapefruit. Plant Growth Regulation, Amsterdam, v. 30, p. 225-232, 2000.

CASTRO, P.R.C. de. Biorreguladores em citros. Laranja, Cordeirópolis, v. 22, n. 2, p. 351-366, 2001.

ERICKSON, L.E. 1968. The general physiology of citrus. In: REUTHER, W.; BATCHELOR, L.D.; WEBBER, H.J. (Eds). The citrus industry. Riverside: University of California, 1968. v. 2. p. 86-122.

GALLASH, P.T. Control of alternate cropping of Valencia orange with etephon and naphthalene acetic acid. Australian Journal of Agricultural and Animal Husbandry, Melbourne, v.18, p.52157,1978

GOLDSCHMIDT, E.E.; GOLOMB, A. The carbohydrate balance of alternate bearing citrus trees and the significance for flowering and fruiting. Journal of American Society for Horticultural Science, Ashford, v. 107, p. 206-208, 1982.

GUARDIOLA, J.L.,MONERRI, C. AGUSTÍ, M. The inhibitory effect of gibberellic acid on flowering in citrus. Physiologia Plantarum, Copenhagen, v. 55, p. 136-142, 1982.

GUARDIOLA, J.L. Utilização de reguladores de crescimento em citricultura. Laranja, Cordeirópolis, v. 15, n. 2, p. 155-177, 1994. 
HIELD, H.Z.; HILGERMAN, R.H. Alternate bearing and chemical fruit thinning of certain citrus varieties. Proceedings International Society of Citriculture, Riverside, v. 3, p. 1145 1153, 1969.

IQBAL, N.; KARACALI, I. Flowering and fruitset behavior of Satsuma mandarin (Citrus unshiu Marc) as influenced by environment. Pakistan Journal of Biological Science, Murree, v. 7, n. 11, p. 1832-1836, 2004.

KOLLER, O.C. Citricultura: laranja, limão e tangerina. Porto Alegre: Rígel, 1994. 446 p.

LORD, E.M.; ECKARD, K.J. Shoot development in Citrus sinensis (Washington navel orange). Alteration of development fat of $\mathrm{GA}_{3}$ treatment. Botanical Gazette, Chicago, v. 148, p. 17-22, 1987.

MARODIN, G.A.B. Raleio químico e manual de frutinhos em tangerineira (Citrus deliciosa Tenore) cv. Montenegrina. 1986. 124f. Dissertação (Mestrado - Programa de Pós-graduação em Fitotecnia) - Faculdade de Agronomia, Universidade Federal do Rio Grande do Sul, Porto Alegre, 1986.

MONSELISE, S.P.; HALEVY, A. H. Chemical inhibition and promotion of citrus flower bud induction. Proceedings of the American Society for Horticultural Science, Alexandria, v. 84, p. 141-146, 1964.

MOSS, G.J. Effect of fruit on flowering in relation to biennal bearing in sweet orange (Citrus sinensis). Journal of Horticultural Science, Ashford, v. 46, p. 177-184, 1971.

MOSS, G.J.; BEVINGTON, K.B. The use of gibberellic acid to control alternate cropping of Late Valencia sweet orange. Australian Journal of Agricultural Research, Melbourne, v. 28, n. 6, p. 1041-1054, 1977.

PEREIRA, I.A.M.; PINTO, J.E.B.; DAVIDE, L.C.Época da indução e evocação em Citrus sinensis (L.) Osbeck cv. Pêra Rio. Ciência Rural, Santa Maria, v. 33, n. 5, p. 857-862, 2003.

RANDHAWA, G.S.; DINSA, H.S. Time of blossom bud differentiation in Citrus. Proceedings of American Society for Horticultural Science, Alexandria, v. 50, p. 1665-1671, 1947.
RODRIGUES, L.R. SCHWARZ, S.F.; RECKZIEGEL, V.P.; KOLLER, O.C. Raleio manual de frutos em tangerineiras 'Montenegrina'. Pesquisa Agropecuária Brasileira, Brasília, v. 33, n. 8, 1998.

SARTORI, I.A. Poda, raleio de frutos e uso de fitorreguladores em tangerineiras (Citrus deliciosa Tenore) cv. Montenegrina. 2005. 99 f. Tese (Doutorado - Programa de Pós-Graduação em Fitotecnia) - Faculdade de Agronomia, Universidade Federal do Rio Grande do Sul, Porto Alegre, 2005.

SCHNEIDER, H. 1968. The anatomy of citrus. In: REUTHER, W.; BATCHELOR, L.D.; WEBBER, H.J. (Eds). The citrus industry. Riverside: University of California, 1968. v.2. p.1-85.

SCHWARZ, S.F. Influência do raleio manual de frutinhos sobre a produção de tangerineira 'Montenegrina' (Citrus deliciosa Tenore). 1989. 106f. Dissertação (Mestrado - Programa de Pósgraduação em Fitotecnia) - Faculdade de Agronomia, Universidade Federal do Rio Grande do Sul, Porto Alegre, 1989.

SCHWARTZ, S.F.; KOLLER, O.C.; NIENOW, A.H. Intensidades e épocas de raleio manual em tangerineiras 'Montenegrina'. Pesquisa Agropecuária Brasileira, Brasília, v. 27, n. 8, p. 1161$1165,1992$.

SOUZA, P.V.D. de. Efeito de concentrações de etefón e pressões de pulverização foliar no raleio de frutinhos em tangerineiras (Citrus deliciosa Tenore) cv. Montenegrina. 1990. $139 \mathrm{f}$. Dissertação (Mestrado - Programa de Pós-graduação em Fitotecnia) - Faculdade de Agronomia, Universidade Federal do Rio Grande do Sul, Porto Alegre, 1990.

TADEO, F.R. et al. Histología y citología de los cítricos. Valencia: Generalitat Valenciana, 2003.99p.

WHEATON, T.A. Alternate bearing. Citrus flowering, fruit set and development. Short Course \& Workshop Proceedings, Flórida, 1986. Disponível em: <www.fcprac.ifas.ufl.edu/> Acesso em: 15 jun. 2005. 